\title{
RESIDÊNCIA EM SAÚDE DA FAMÍLIA DO CAMPO E O ENFRENTAMENTO A COVID-19: RELATO DE EXPERIÊNCIA
}

\section{FAMILY HEALTH RESIDENCE IN CAMPO AND COPING WITH COVID-19: EXPERIENCE REPORT}

\author{
Maria Deisyelle Sibaldina Da Almeida ${ }^{1}$ \\ Fellype Ribeiro da Silva Psicólogo 2 \\ Nicole Caroline Nascimento da Silva ${ }^{3}$ \\ Priscilla Maria de Assumpção Costa Ferreira Enfermeira ${ }^{4}$ \\ Emmanuelly Correia de Lemos $^{5}$ \\ Itamar Lages $^{6}$
}

1- Profissional de Educação Física. Residente do Programa de Residência de Saúde da Família com ênfase nas Populações do Campo/UPE, Garanhuns, Brasil.

2- Residente do Programa de Residência de Saúde da Família com ênfase nas Populações do Campo/UPE, Garanhuns, Brasil.

3- Profissional de Educação Física. Residente do Programa de Residência de Saúde da Família com ênfase nas Populações do Campo/UPE, Caruaru, Brasil.

4- Residente do Programa de Residência de Saúde da Família com ênfase nas Populações do Campo/UPE, Caruaru, Brasil.

5- Coordenadora de Educação Permanente em Saúde da Escola de Governo em Saúde Pública de Pernambuco, Secretaria Estadual de Saúde de Pernambuco, Pernambuco, Brasil. Mestre em Saúde Pública/FIOCRUZ-PE. Doutora em Educação Física/UPE, Recife, Brasil.

6- Enfermeiro Sanitarista. Mestre em Saúde Coletiva. Professor da Universidade de Pernambuco. Coordenador do Programa Multiprofissional de Residência em Saúde da Família do Campo da 
UPE. Professor do Curso de Graduação em Enfermagem da FENSG-UPE. Membro do Centro

Brasileiro de Estudos de Saúde.

\title{
RESUMO
}

A pandemia da COVID-19 é a principal causa de mudança e adaptação em todos os níveis de atenção à saúde. Com isso, o objetivo deste estudo foi descrever as ações desenvolvidas pelas Equipes de Saúde da Família do Campo no contexto da COVID-19 a partir das vivências de residentes. Trata-se de um estudo descritivo, tipo relato de experiência, realizado no período de março a maio de 2020. Utilizou-se os registros dos diários de campo e portfólios dos residentes e foram criadas três categorias analíticas para sistematização das experiências. Foi evidenciado que para manter as ações da atenção primária no contexto da COVID-19, foram pontos chaves: reuniões sistemáticas de equipe, educação permanente, atuação dos residentes do campo e a utilização de diferentes recursos para comunicação. Com isso, é essencial no enfrentamento da atual pandemia, necessitando-se fazer desta uma forte protagonista neste momento de fortalecimento do modelo universal, integral, equânime e interprofissional.

Palavras-chaves: Coronavírus. Atenção Primária à Saúde. População Rural. Assistência integral à saúde. Equipe Multiprofissional.

\begin{abstract}
The COVID-19 pandemic is the main cause of change and adaptation at all levels of health care. Thus, the objective of this study was to describe the actions developed by the Family Health Teams of the Countryside in the context of COVID-19 from the experiences of residents. This is a descriptive study, type of experience report, carried out in the period of March to May 2020. The records of the residents' field diaries and portfolios were used and three analytical categories were created to systematize the experiences. It was evidenced that in order to maintain the actions of primary care in the context of COVID-19, key points were: systematic team meetings, permanent education, performance of rural residents and the use of different resources for communication. With this, it is essential to face the current pandemic, making it necessary to make it a strong protagonist in this moment of strengthening the universal, integral, equitable and interprofessional model.
\end{abstract}

Keywords: Coronavirus. Primary Health Care. Rural Population. Comprehensive health care. Multiprofessional team. 


\section{INTRODUÇÃO}

Nos últimos meses, o Brasil está vivendo com a emergência de uma complexa situação envolvendo uma doença que tem alto teor de contágio. A epidemia da COVID-19que se iniciou em Hubei, na China, se disseminou rapidamente por outros países, sendo declarado pela Organização Mundial de Saúde (OMS) situação de pandemia em pouco mais de dois meses desde o início ${ }^{1}$.

Logo, com o desenrolar da pandemia, o sistema público de saúde no Brasil passa a ter que se prepararpara dar conta de uma situação extremamente complicada visto as diversas iniquidades que envolve o financiamento, gestão e estruturação em que o SUS se encontra. Nesse sentido, as Equipes do Programa Saúde da Família (PSF) e dos Núcleos de Apoio à Saúde da Família (NASF), enquanto operadoras da atenção primária à saúde (APS), precisaram encontrar mecanismos para acolher e dar encaminhamentos de resolutivos aos problemas decorrentes dessa situação.E essas Equipes têm que fazer isso porque lhes cabe a responsabilidade sanitária por um território, a permanente construção de vínculos e a coordenação do cuidado pela qual busca realizar a integralidade da assistência e da vigilância à saúde. Desse entendimento decorre o principal postulado deste estudo: a APS praticada pelas Equipes de PSF e do NASF são fundamentais para resolver o problema da transmissão, adoecimento e, morte prematura pela COVID-19 ${ }^{1,2}$.

Compreendendo o papel da APS na pandemia da COVID-19, ela se torna ainda mais relevante para populações que historicamente foram marcadas, por um processo de marginalização e ausência de políticas públicas, como as populações do campo. Por outro lado, com a institucionalização da Política Nacional de Saúde Integral das Populações do Campo e da Floresta e Águas ${ }^{3}$ vem sendo possível vivenciar algumas conquistas para essas populações. Uma delas foi a implantação de Unidades e Equipes de Programa Saúde da Família que são acompanhadas por Equipes do Núcleo de Apoio à Saúde da Família. 
Para contribuir com a Política acima mencionada, um grupo de docentes da Universidade de Pernambuco e militantes de entidades como o Movimentode Trabalhadores Rurais Sem Terra e da Associação Quilombola de Castainho criou o Programa de Residência Multiprofissional em Saúde da Família com Ênfase na Saúde do Campo (REMSFC) para formar sujeitos comprometidos com o confronto à desigualdade social em saúde e com a materialização dos princípios do Sistema Único de Saúde (SUS) por meio de um instrumental técnico capaz de elevar a qualidade assistencial e da vigilância à saúde, de modo a propiciar acesso às ações e serviços de saúde ${ }^{4}$.

A equipe de residentes da REMSFC é composta pelas seguintes categorias profissionais: Educação Física, Enfermagem, Farmácia, Fisioterapia, Fonoaudiologia, Nutrição, Odontologia, Psicologia, Terapia Ocupacional, Saúde Coletiva e Serviço Social. A Equipe selecionada por procedimentos públicos é distribuída em dois grupos de dez pessoas representantes das categorias acima citadas nos territórios rurais com e sem assentamentos de reforma agrária e quilombos dos municípios de Caruaru e Garanhuns.

Com a pandemia da COVID-19, a assistência à saúde dessas populações passa a ser uma questão muito delicada e que exige uma atenção específica e cautelosa das Equipes de PSF e do NASF, pois é sabido que o vírus da doença tem alta transmissibilidade e provoca uma síndrome respiratória aguda que varia de casos leves a casos muito graves com insuficiência respiratória que pode evoluir para o óbito.

Contudo, apesar das inúmeras dificuldades que o SUS enfrenta, bem como a urgente redefinição do modelo de atuação na atenção primária ${ }^{5}$ cujos efeitos negativos se verificam na atual situação de epidemia reafirma-se que de fundamental importância disparar processos de reflexão a respeito das possibilidades de gerir a atenção multiprofissional diante desse momento histórico de maneira a não desassistir as populações. 
Nesse sentido, considerando o misto de vivências provocadas pelo contexto da pandemia da COVID-19 para Equipes do PSF do Campo em conjunto com a da REMSFC é que surge a motivação para escrita desse estudo, em formato de relato de experiência, que tem como objetivo descrever as ações desenvolvidas pelas Equipes dos PSF do Campo no contexto da COVID-19 a partir das vivências de residentes da REMSFC.

\section{MATERIAL E MÉTODOS}

Trata-se de um estudo descritivo, tipo relato de experiência, que possibilita a descrição e reflexão sobre um determinado fenômeno ${ }^{6}$, referente às ações dos residentes da Residência em Saúde da Família com ênfase nas Populações do Campo, enquanto estratégia multiprofissional de enfrentamento a pandemia do COVID-19 nos territórios de abrangência.

O estudo foi realizado nas localidades onde a quinta turma da REMSFC está em atuação, ou seja, naVila Rafael e em Cachoeira Seca, zona rural do município de Caruaru; e nos quilombos rurais de Estivas, Castainho e Tigre, em Garanhuns. Todas, situadas no estado de Pernambuco.

O período do estudo foi compreendido de março a maio de 2020, onde foram realizadas estratégias de enfrentamento a COVID-19 e implementadas na comunidade para a prevenção, promoção e recuperação da saúde dessas populações, buscando contribuir para uma assistência à saúde integral e multidisciplinar.

Para a realização deste relato, foram utilizados materiais que fazem parte da estrutura organizacional da REMSFC que se constituem como sistematizações do processo de ensino aprendizagem, que são os portfólios e diários de campo, sendo elaboradas a partir disso, categorias de análise e discussão, a saber: a pandemia da COVID-19 chega ao território das Equipes e da Residência de Saúde da Família do Campo, Mudança na rotina de trabalho das Equipes e as ações da Residência de Saúde da Família do Campo no território frente à pandemia 
e Percepção e adesão da comunidade as ações das Equipes dos PSF e da Residência de Saúde da Família do Campo no território frente à pandemia.

Para compor o escopo teórico dessas ações de saúde, buscou-se artigos científicos que abordassem o tema em questão e estivessem indexadas em bases dados como Scielo e Biblioteca Virtual da Saúde, com recorte de 2016 a 2020, utilizando como descritores as seguintes palavras-chave: Atenção Primária à Saúde, Assistência Integral à Saúde, População Rural, Equipe Multiprofissional, Coronavírus, Sistema Único de Saúde.

O presente estudobuscousalvaguardar os aspectos éticos através do anonimato, bem como quais atividades se restringem a uma ou outra USF e comunidade, garantindo o uso das informações apenas para fins acadêmicos, respeitando as recomendações do Conselho Nacional de Ética em Pesquisa.

\section{RESULTADOS E DISCUSSÃO}

A partir dos registros nos portifólios e diário de campo dos residentes da REMSFC foram construídas as sistematizações abaixo descritas a partir de três categorias julgadas imprescindíveis para a compreensão das experiências vividas em conjunto pela Equipe da residência e do PSF na assistência à saúde das populações tradicionais como do campo no contexto da COVID-19.

\section{A pandemia da COVID-19 chega ao território das Equipeseda Residência de Saúde da}

\section{Família do Campo}

O Ministério da Saúde reconheceu e declarou, por meio da portaria nº 454 de março de 2020, que todo o território nacional se encontrava no estado transmissão comunitária do vírus. Este estado de transmissão é bastante rápido e não é possível mais identificar os transmissores 
do vírus, com isso a APS entrou em estado de alerta para que pudesse notificar e acompanhar os casos sintomáticos leves da doença ${ }^{7}$.

Diante desse cenário, a rotina de trabalho de todos que estão na APS se modificou, assim foi necessário que toda a equipe estivesse capacitada, compreendendo o fluxograma de manejo clínico na atenção primária em transmissão comunitária ${ }^{7}$. Para isso, foram necessárias reuniões de equipe e educação permanente, onde a residência pode contribuir fortemente nesse processo.

A equipe do PSF e da REMSFC se organizaram de modo a atender apenas urgência, consultas de pré-natal, renovação de medicações e estruturou outros processos para estabelecer comunicação com a população, que serão explanados mais à frente. Integrantes da REMSFC também precisaram estabelecer um rodízio durante a semana de modo a diminuir quantidade de pessoas nas unidades.

A equipe foi formada por meio de reuniões para organizar o fluxo, sem movimentação de pacientes pelos corredores da unidade. Os usuários, para evitar contato, foram orientados a distância a ser mantida um dos outros, principalmente em tempo de campanha de vacina que a quantidade de pessoas é maior. Todos os idosos foram vacinados em casa para não haver pessoas de grupo de risco circulando nas ruas. O material sobre o manejo clínico foi usado para triagem dos usuários que chegavam e para ao mesmo tempo aconselhá-los das medidas preventivas. Os pacientes sintomáticos foram direcionados a lugares mais ventilados. O suporte psicológico também foi acionado para os pacientes que apresentarem necessidade desse serviço.

Outro fator que gerou preocupação foi a pequena quantidade de equipamentos de proteção individual (EPI) disponibilizada pelas Secretarias de Saúde, pois esse fator gera insegurança e medo nos profissionais quando se considera a elevada capacidade de transmissão do vírus e, consequentemente, adoecimento e mortes. E essas preocupações são justificadas pela leitura de notícias que dão conta do elevado quantitativo de mortes dos profissionais de 
saúde ${ }^{8}$. Por outro lado, é sabido que o mercado de EPI's se tornou um objeto de disputa geopolítica no qual as Secretarias Municipais e Estaduais têm pouquíssima influência?

Vale destacar que pela característica de proximidade que as Equipes de PSF tem com a população foi possível visualizar e pensar os protocolos compreendendo e aproximando das realidades vividas pela população do campo acompanhada. Pois, como afirma Sartiet al. " "a APS é potente na redução das iniquidades em saúde e deve, portanto, ser fortalecida e estruturada como uma das principais respostas do setor saúde à epidemia" ao considerarmos o alcance e a proximidade com as diversas iniquidades que a população vivencia.

\section{Mudança na rotina de trabalho das Equipes e as ações da Residência de Saúde da Família do Campo no território frente à pandemia}

No primeiro trimestre de 2020, quando a pandemia emergiu fortemente no Brasil, a REMSFC iniciou de imediato ações de educação em saúde, principalmente nos espaços de sala de espera dos PSF, com o objetivo de alertar e informar a população a respeito daCOVID-19, ressaltando principalmente as principais medidas de prevenção orientadas pelo Ministério da Saúde.

A partir do mês de março o atendimento nas unidades de PSF ficou restrito a renovação de medicação, acompanhamento de gestantes e visitas domiciliares restritas, e atendimentos considerados de urgência para diminuir a aglomeração de pessoas por orientação das Secretarias Municipais de Saúde. Atrelado a isso, o uso de EPI's passou a ser estritamente indispensável em qualquer atendimento a ser realizado.

Com as necessárias mudanças na rotina das Equipes dos PSF, foi preciso planejar outras formas de comunicação com a população, para além dos atendimentos de urgência. Assim, foi necessário recorrer a outros meios de comunicação via celular para facilitar o acesso às informações e cuidado contínuo da saúde dos/as usuários/as. A grande maioria dos contatos 
estabelecidos foram realizados por meio de aplicativo de conversa de celular, por ser uma ferramenta que a maioria da população tem acesso, onde as pessoas podem tirar dúvidas e receber orientações.

A visita domiciliar, e mais ainda o teleatendimento, passaram a ser as ferramentas tecnológicas mais utilizadas para o atendimento. A segunda foi a utilizada pelos residentes. Segundo Sartiet al. ${ }^{1}$ a adequada implantação de tecnologias aumenta a capacidade de atendimento do sistema de saúde, facilita o acesso das pessoas a orientações qualificadas, contribui para a redução da sobrecarga da APS e outros níveis de atenção, e auxilia no ordenamento do fluxo das pessoas no sistema.

Os Agentes Comunitários de Saúde também se encontravam com limitações para a realização de rotina das visitas domiciliares, sobretudo devido à escassez de EPI's. No entanto, estes continuaram a exercer um papel fundamental no intermédio de comunicação entre a comunidade e o PSF.

Ainda, em meio à pandemia do COVID-19 algumas ações como o calendário de Campanha Nacional de Vacinação da Influenza 2020 sofreu alterações, de acordo com as orientações do Ministério da Saúde, iniciando em março com previsão de se estender até o início de junho, respeitando o cronograma ministerial, sendo realizadas a vacinação porta-aporta, para evitar aglomeração, somente as crianças e gestantes se dirigiram à unidade, onde cada microárea tinha seu dia específico para a vacinação, respeitando as medidas de segurança preconizadas.

Para que os residentes pudessem contribuir ainda mais com as ações da USF, estes realizaram cursos virtuais disponibilizados pelo UNA-SUS sobre a doença COVID-19, a sua prevenção e manejo na APS. A partir desses saberes construídos, foi possível planejar mais ações e estruturar projetos que pudessem focar na realidade das comunidades atendidas. 
A abordagem da Equipe do PSF e da REMSFC possibilitou pensar em ações estratégicas conjuntas por diversos profissionais que têm um objetivo em comum, proteger e diminuir os efeitos da pandemia do COVID-19 no território ${ }^{10}$. Ações como distribuição de máscaras, educação popular em saúde foram feitas para minimizar riscos à saúde.

Além disso, a produção de vídeos educacionais visando a disseminação de informações relacionadas ao autocuidado (ex.: realização de exercícios físicos em casa), manuseio e higienização das máscaras, orientações de como lavar as mãos também foram desenvolvidos e compartilhados na comunidade. A criação de canais remotos por meio de telefone e videoconferência foram as estratégias mais usadas para os atendimentos e dúvidas dos usuários. Os atendimentos via aplicativo de celular foram divididos pelos médicos dos PSF e, se necessário, evoluiriampara um agendamento de consulta presencial.

A elaboração de um projeto intitulado Quilombo com saúde: COVID-19, não! foi criado com o objetivo de provimento de água e máscaras para as comunidades do campo. Esse projeto respondeu ao edital da Fundação Oswaldo Cruz para formação de uma rede de parcerias contra o COVID-1911.

Os residentes trabalharam junto com a equipe dos PSF na separação dos nomes de usuários mais de 60 anos, domiciliados e acamados para o Programa Remédio na Porta desenvolvido pela Secretaria de Saúde de Caruaru com o objetivo de garantir a entrega de medicamentos de uso contínuo para usuários que possuem doenças crônicas, isso durante a pandemia, podendo ser estabelecida como prática padrão após esse período.

A equipe da residência também atuou na realização de educação permanente sobre o Sistema Único de Assistência Social (SUAS) para a compreensão de vários serviços ofertados, inclusive sobre o auxílio emergencial e sobre o uso irracional dos medicamentos.

Essas propostas foram desenvolvidas para um atendimento seguro e resolutivo em diversos aspectos, permitindo a diminuição de contaminação e ser um canal de educação em 
saúde. Além desses processos, pensando na saúde do trabalhador, e compreendendo o momento delicado e de medo que os profissionais de saúde enfrentavam, foram realizadas algumas propostas de cuidado voltadas para equipe dos PSF. Assim, os residentes ofertaram cuidado por meio de algumas práticas integrativas e complementares.

A partir do exposto foi possível verificar que a APS para dar conta de atender às demandas trazidas pela Covid-19, precisou recuperar seus princípios de base comunitária, trabalho em equipe, intra e extramuros, com usuários, pacientes, famílias e comunidade ${ }^{12}$ reflexões amplificadas neste período de pandemia que precisarão de atenção quanto aos desdobramentos pós pandemia.

\section{Percepção e adesão da comunidade as ações das Equipes e da Residência de Saúde da Família do Campo no território frente à pandemia}

Mesmo com todos os cuidados tomados pelas Equipes de PSF e da REMSFC, mudar uma rotina que há anos estava estabelecida, foi muito difícil no início, pois os usuários precisaram se adaptar aos novos fluxos que foram criados e recriados a partir das recomendações do Ministério da Saúde e Organização Mundial da Saúde.

Diversas reuniões de equipe, diálogos e ajustes precisaram serem realizados para que a comunidade não deixasse de ser assistida em suas necessidades, onde foi de grande importância a atuação da REMSFC na articulação das estratégias discutidas nas reuniões, bem como na organização dos fluxos nas unidades de saúde e orientação da população.

A nova dinâmica que a pandemia da COVID-19 nos apresentou, deveras desafiadora, pois os usuários ainda se encontravam resistentes em adotar medidas de segurança e muitos vinham desprotegidos a USF, somente com o diálogo e entendendo o contexto individual e coletivo, foi possível desenvolver as ações e ter a adesão da comunidade. 
A reestruturação das condutas nas USF foram estratégias positivas no enfrentamento a COVID-19, porém a quantidade insuficiente de EPI's, foi uma situação negativa, onde trabalhávamos com os materiais restringidos e não podíamos ofertá-los a comunidade.

Diante de todas as estratégias elaboradas e postas em prática nos territórios, é importante destacar que elas só puderam ser viabilizadas graças a uma ferramenta de grande força motriz e que transforma ações em reações efetivas, contribuindo para um processo de saúde adequado a APS, que é a Educação Popular em Saúde, materializada na Política Nacional de Educação Popular em Saúde no Sistema Único de Saúde ${ }^{13}$.

A educação popular em saúde é caracterizada como uma construção coletiva onde o facilitador e a comunidade colaboram mutuamente, com objetivo de educar para a saúde, evitando adoecimentos e agravos a saúde, esse foi o caminho para instrumentalização da comunidade e estímulo a autonomia ${ }^{14,15}$.

\section{CONSIDERAÇÕES FINAIS}

As vivências na APS em meio a uma pandemia de COVID-19 evidenciaram arelevância da organização oportuna das Equipes, sendo chave as reuniões sistemáticas de planejamento estratégico, para uma adequada reorganização do serviço frente a sua realidade. Como também que a educação permanente em saúde se configurou como caminho efetivo para a promoção de um cuidado mais resolutivo, produzir uma maior segurança aos profissionais durante sua atuação.

Foi também explicitado, que em tempos de crise como o atual, trabalhar em equipe multiprofissional, em especial com equipe de residentes, além de cumprir os objetivos constitucionais quanto a formação de recursos humanos para o SUS, também possibilitou a construção de diferentes ações de cuidado no território, desde a reorganização dos atendimentos no PSF até o desenvolvimento de outras formas de comunicação e de ofertas de atividades aos 
usuários/as dentro das singularidades da vida do campo. Sendo destacado aqui, a Educação Popular em Saúde, como chave para um processo de cuidado integral.

Com isso, é importante reafirmar a importância fundamental das práticas de APS pelas Equipes profissionais e da REMSFC no enfrentamento da COVID-19 e na superação do modelo biomédico. Desse modo, ressaltamos a potência das equipes multiprofissionais, pois com elas são abertas e ampliadas as possibilidades de promoção, proteção e recuperação da saúde para resolver os problemasque atravessam negativamente o necessário processo de otimização da saúde e da autonomia para que seja possível viver de maneira participativa, livre e crítica. 


\section{REFERÊNCIAS}

1. SARTI, TD, LAZARINI WS, FONTENELLE LF, et al. Qual o papel da Atenção Primária à Saúde diante da pandemia provocada pela COVID-19? Epidemiol. Serv. Saude, 2020. 29(2):15, [acesso em 2020 mai 15] Disponível em: https://www.scielosp.org/article/ress/2020.v29n2/e2020166/

2. PEREIRA PAP. Necessidades humanas. Subsídios à crítica dos mínimos sociais. São Paulo: Cortez, 215p, 2000.

3. BRASIL, Ministério da Saúde. Portaria $\mathrm{n}^{\circ}$ 2.866, de 2 de dezembro de 2011. Aprova a Política nacional de saúde integral das populações do campo e da floresta. Brasília, Distrito Federal, Brasil. 2 de dez 2011.

4. PERNAMBUCO UPE. Projeto Político e Pedagógico do Programa de Residência Multiprofissional em Saúde da Família com Ênfase na Saúde da População do Campo "Lato Sensu". [acesso em 2020 mai 30] Disponível em: https://drive.google.com/file/d/1GNDyfyLx9fdaVpwIeKKahMqcgfE1uNjP/view?usp=sharin $\mathrm{g}$

5. MOROSINI MVGC, FONSECA AF. Revisão da Política Nacional de Atenção Básica numa hora dessas? Cad. Saúde Pública. 2017. 33(1):1-5. [acesso em 2020 mai 15] Disponível em: http://www.scielo.br/pdf/csp/v33n1/1678-4464-csp-33-01-e00206316.pdf.

6. MINAYO MCS, MINAYO-GOMEZ C. Difíceis e necessárias relações entre métodos quantitativos e qualitativos. 2003. Organizadores Books: Goldenberg P, Marsiglia RMG, Gomes MHA. O clássico e o novo: tendências, objetos e abordagens em ciências sociais e saúde. Editora Fiocruz, 2003. [acesso em 2020 mai 15] Disponível em: $\underline{\text { http://books.scielo.org/id/d5t55/09 }}$ 
7. BRASIL, Ministério da Saúde. Protocolo de Manejo Clínico do Coronavírus (COVID-19) na Atenção Primária à Saúde - Versão 9. Brasília, 2020. [acesso em 2020 mai 15] Disponível em: https://portaldeboaspraticas.iff.fiocruz.br/wpcontent/uploads/2020/05/20200504_ProtocoloManejo_ver09.pdf

8. Confederação dos trabalhadores do serviço público federal. Mundo registra 260 profissionais de saúde mortos por Covid-19, 130 só no Brasil. Publicado em 19 de maio de 2020. [acesso em 2020 mai 22] Disponível em: https://www.condsef.org.br/noticias/mundo-registra-260profissionais-saude-mortos-por-covid-19-130-so-brasil

9. CNN Brasil. Pandemia de coronavírus desencadeia disputa global por máscaras de proteção. Publicado em 05 de abril de 2020. [acesso em 2020 mai 22] Disponível em: https://www.cnnbrasil.com.br/internacional/2020/04/05/pandemia-de-coronavirus$\underline{\text { desencadeia-disputa-global-por-mascaras-de-protecao }}$ 10. GUIMARÃES ASM, CUNHA TGS, SANTOSET TA, et al. Atuação da equipe multiprofissional em saúde, no cenário da pandemia por Covid 19. Health Residencies Journal, 2020. 1(2)1-22. [acesso em 2020 mai 22] Disponível em:

$\underline{\text { https://escsresidencias.emnuvens.com.br/hrj/article/view/37 }}$

11. FIOCRUZ. Fiocruz lança programa de parcerias contra para ações contra a COVID-19. Publicado em 03 de abril de 2020. Brasil. [acesso em 2020 mai 22] Disponível em: https://portal.fiocruz.br/noticia/fiocruz-lanca-programa-de-parcerias-para-acoes-contra-covid$\underline{19}$

12. NEDEL FB. Enfrentando a COVID-19: APS forte agora mais que nunca! APS em revista, 2020. 2(1)11-16. [acesso em 2020 mai 25] Disponível em: https://apsemrevista.org/aps/article/view/68 
13. ROCHA MB. Panorama sobre o saber e a experiência popular nas práticas de Educação em Saúde: uma revisão integrativa. Anais do $16^{\circ}$ Congresso Brasileiro de Assistentes Sociais, 2019. [acesso em 2020 mai 25] Disponível em: http://broseguini.bonino.com.br/ojs/index.php/CBAS/article/view/1899/1855

14. BRASIL, Ministério da Saúde. Portaria no 2.761, de 19 de novembro de 2013. Institui a Política Nacional de Educação Popular em Saúde no âmbito do SUS. Brasília, Brasil. 2013. [acesso em 2020 mai 25] Disponível em: http://www.crpsp.org.br/diverpsi/arquivos/PNEPS$\underline{\text { 2012.PDF }}$

15. STHAL H, LEAL C. Educação Popular como Política de Saúde: interfaces com a formação profissional em saúde. Cad. Pes., São Luís. 2017. 24(2)125-138. [acesso em 2020 mai 25] Disponível em: http://www.periodicoseletronicos.ufma.br/index.php/cadernosdepesquisa/article/view/7518/46 $\underline{32}$ 